\title{
Fold-change detection in a whole-pathway model of Escherichia coli chemotaxis
}

Article

Accepted Version

Edgington, M. P. and Tindall, M. J. (2014) Fold-change detection in a whole-pathway model of Escherichia coli chemotaxis. Bulletin of Mathematical Biology, 76 (6). pp. 13761395. ISSN 0092-8240 doi: https://doi.org/10.1007/s11538014-9965-3 Available at https://centaur.reading.ac.uk/36761/

It is advisable to refer to the publisher's version if you intend to cite from the work. See Guidance on citing.

To link to this article DOI: http://dx.doi.org/10.1007/s11538-014-9965-3

Publisher: Springer

All outputs in CentAUR are protected by Intellectual Property Rights law, including copyright law. Copyright and IPR is retained by the creators or other copyright holders. Terms and conditions for use of this material are defined in the End User Agreement.

\section{www.reading.ac.uk/centaur}

\section{CentAUR}

Central Archive at the University of Reading

Reading's research outputs online 


\title{
Fold-change detection in a whole-pathway model of Escherichia coli chemotaxis
}

Received: date / Revised: date

\begin{abstract}
There has been recent interest in sensory systems that are able to display a response which is proportional to a fold-change in stimulus concentration, a feature referred to as fold-change detection (FCD). Here we demonstrate FCD in a recent whole-pathway mathematical model of Escherichia coli chemotaxis. FCD is shown to hold for each protein in the signalling cascade and to be robust to kinetic rate and protein concentration variation. Using a sensitivity analysis we find that only variations in the number of receptors within a signalling team lead to the model not exhibiting FCD. We also discuss the ability of a cell with multiple receptor types to display FCD and explain how a particular receptor configuration may be used to elucidate the two experimentally determined regimes of FCD behaviour. All findings are discussed in respect of the experimental literature.
\end{abstract}

Keywords Bacterial chemotaxis · Signalling cascade · Adaptation

\section{Introduction}

Chemotactic bacteria such as Escherichia coli swim via a series of runs and tumbles [33], producing random walk type behaviour. Runs are associated with counter-clockwise rotation of a cells' 4 to 6 flagella [34] which propel the cell forward. This differs from tumbles which are caused by clockwise flagella rotation acting to randomly reorient the cell. The direction of movement is altered a least once every few seconds [37]. Cells are able to move to areas with favourable conditions by increasing the run time when moving up an attractant gradient thus biasing their movement in response to the sensing of chemical gradients [1].

E. coli cells possess five different types of chemoreceptors [10], four of which (Aer not included) are known as methyl-accepting chemotaxis proteins (MCPs), which are able to detect chemoaffectors (attractants or repellents). MCPs are linked to the flagellar motors by an intracellular signalling pathway as shown in Figure 1. Chemoreceptors associate with a linker protein CheW and the histidine kinase CheA. In the absence of an attractant gradient CheA autophosphorylates (CheA-P) [37]. Phosphoryl groups are transferred from CheA-P to either the response regulator CheY or the methylesterase

M.P. Edgington · M.J. Tindall

Department of Mathematics \& Statistics, University of Reading, Whiteknights, PO Box 220, Reading, RG6 $6 \mathrm{AX}, \mathrm{UK}$.

Tel.: +440118 3788992

Fax: +4401189313423

E-mail: m.tindall@reading.ac.uk

M.J. Tindall

School of Biological Sciences, University of Reading, Whiteknights, Reading, RG6 6BX, UK.

M.J. Tindall

Institute for Cardiovascular and Metabolic Research, University of Reading. PO Box 218, Whiteknights, Reading, RG6 6AA, UK. 


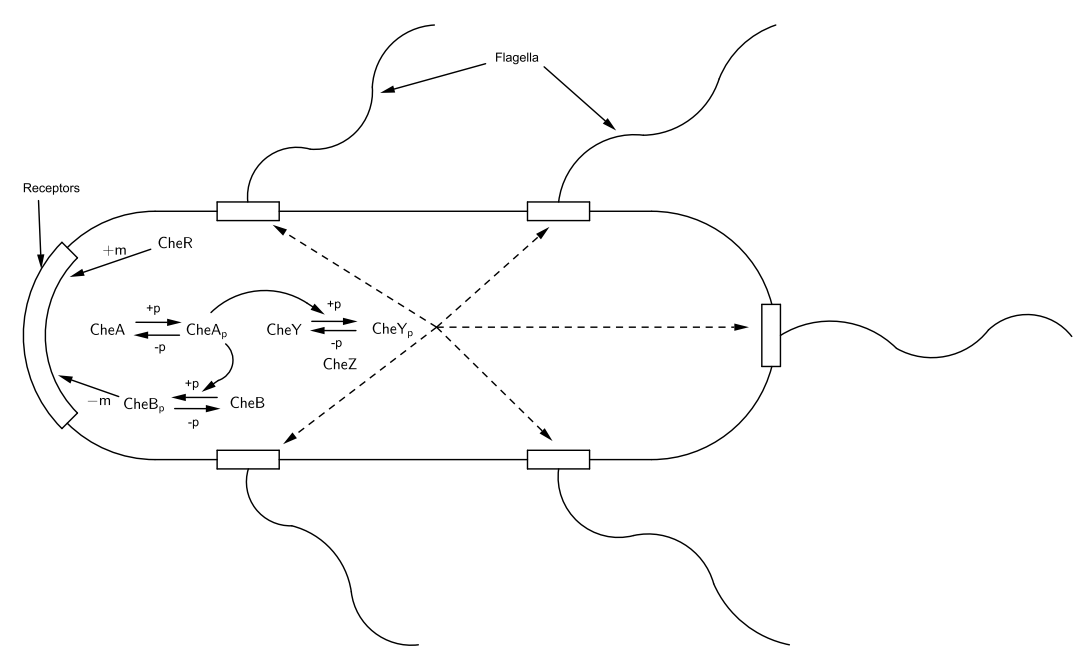

Fig. 1 A schematic representation of the intracellular signalling pathway in E. coli chemotactic bacteria. Solid lines represent reactions relating to phosphorylation and methylation whilst dashed lines indicate protein diffusion. The labels $+\mathrm{m}$ and $-\mathrm{m}$ refer to the addition and removal of methyl groups to/from receptors, respectively. Similarly $+p$ and $-p$ represent the addition and removal of phosphoryl groups to/from chemotaxis proteins. Cell not drawn to scale.

CheB. Phosphorylated CheY (CheY-P) is then able to diffuse within the cell cytoplasm towards the flagellar motors $[20,4]$. Once the CheY-P has reached the flagella motor it can bind to the motor switching protein FliM causing a greater bias toward tumbling [38]. In E. coli a phosphatase CheZ acts to increase the rate of dephosphorylation of CheY-P [19]. Phosphorylated CheB (CheB-P) along with the methyltransferase CheR forms the adaptation component of the signalling cascade, re-setting the receptors to their pre-stimulus state. CheR acts to methylate the receptors, increasing their activity [31] whilst CheB-P demethylates them, decreasing their activity [32].

Upon detection of an attractant gradient CheA autophosphorylation is reduced and the levels of CheY-P and CheB-P fall. Reduced CheY-P levels cause the flagellar motors to rotate counterclockwise inducing a chemotactic run, whilst a reduction in CheB-P allows the receptors to become methylated given the constant methylating action of CheR. This leads to a return to pre-stimulus levels of CheA autophosphorylation and thus those of CheY-P and CheB-P. The ability of E. coli cells to return themselves to a pre-stimulus state (via adaptation) is one of the key factors enabling them to remain responsive over the observed five orders of magnitude in ligand concentration [22].

Fold-change detection (FCD) has, in recent years, become a popular area of scientific research. It is defined as the property whereby a sensory system will display exactly the same amplitude, shape and recovery time in response to any equal fold-change in input relative to the background concentration stimulus [29] (as shown in Figure 2). This property was hinted at in the early 1970s when it was suggested that $E$. coli gave a similar response to fractional increases in ligand concentration [23]. Recent experimentation using in vivo fluorescence resonance energy transfer (FRET) techniques has now confirmed, using measurements of the adaptation time of cells, that E. coli do in fact display FCD $[16]$.

It has been suggested that adopting an FCD strategy may be beneficial to chemotactic bacteria; the process of a sensory search involving movement through a field becomes invariant to the amplitude of the field itself [29]. This allows the bacterium to pick out scalar multiples in the extracellular environment and therefore focus on the characteristic shape of the chemical field. This suggestion was then built upon with the idea that this sensory search may well be optimal in cases where discovery of any nutrient source is of benefit [16].

Previous work has shown that FCD will be evident in a simplified model of the E. coli chemotaxis signalling pathway [29] as proposed by Tu et al. [35]. This model takes a Monod-Wyman-Changeux 
(a)

(c)
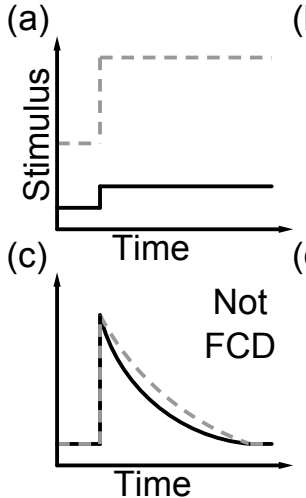

(b)

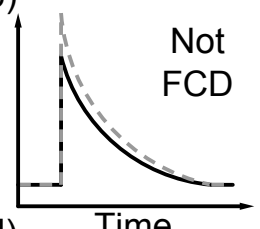

(d)

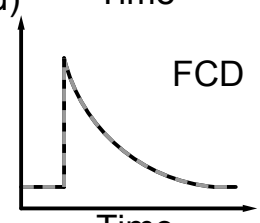

Fig. 2 After [29], a graphical representation of the definition of FCD. The three responses (b)-(d) are possible given the input stimulus (change in ligand concentration) (a). Figure (d) shows two responses which are exactly the same in terms of response amplitude, shape and recovery time. Figure (b) shows responses with different amplitude and (c) a different response shape.

(MWC) representation of receptor clustering and couples it with two ordinary differential equations (ODEs) describing the receptor methylation level and CheY-P concentration.

In this manuscript we consider whether a more detailed model of the signalling dynamics in E. coli will also display FCD. The model considered here couples an MWC description of receptor clustering with ODEs for each of the key chemotaxis signalling proteins. This model is examined using both both theoretical and numerical techniques. A sensitivity analysis is used to show that FCD is invariant to changes in all parameters affecting the signalling cascade so long as they remain constant in time. Consideration is then given to our ability to observe FCD in models comprising multiple receptor types. The biological feasibility of conditions imposed in order to obtain FCD for each receptor configuration is discussed in light of experimental data.

\section{Conditions for FCD}

In this section we outline the theoretical conditions required for a mathematical model of the form

$$
\begin{aligned}
& \dot{x}=f(x(m), \Phi,[L]), \\
& \Phi=g(x(m), \Phi,[L]),
\end{aligned}
$$

to exhibit FCD as recently shown by Shoval and colleagues [29,28]. Here $\Phi$ denotes a receptor signalling team's activity, $[L]$ is the extracellular ligand concentration and $x(m)$ is defined as an effective (methylation dependent) dissociation constant.

A set of four sufficient conditions are given for the existence of FCD [29,28]. The first two of these are that the model must exhibit a stable steady-state and exact adaptation. In addition to this a pair of homogeneity conditions are given relating to a model of the form displayed in equations (1) and (2), namely

$$
\begin{aligned}
& f(p x(m), \Phi, p[L])=p f(x(m), \Phi,[L]), \\
& g(p x(m), \Phi, p[L])=g(x(m), \Phi,[L]),
\end{aligned}
$$

in which $p$ is some constant such that $p>0$. A proof of these two conditions as well as a generalisation allowing a function $\varphi(p, x)$ to be used in place of a constant $p$ are given in the supplementary information of [29].

\section{A model of the E. coli signalling pathway}

We now progress to examining whether a whole-pathway mathematical model of intracellular signalling in E. coli, as recently proposed by Clausznitzer and colleagues [5], can exhibit FCD. The model considers the intracellular chemotactic signalling cascade listed in Table 1 . The model is a more complete 
Table 1 Reactions included within the Clausznitzer et al. model and the rate constants associated with them $[5]$.

\begin{tabular}{cll}
\hline Reaction & Definition & Rate (Reverse) \\
\hline $\mathrm{A} \stackrel{k_{A}}{\longrightarrow} \mathrm{A}-\mathrm{P}$ & CheA autophosphorylation & $10 \mathrm{~s}^{-1}$ \\
$\mathrm{~A}-\mathrm{P}+\mathrm{Y} \stackrel{k_{Y}}{\longrightarrow} \mathrm{A}+\mathrm{Y}-\mathrm{P}$ & CheY phosphorylation & $100 \mu \mathrm{M}^{-1} \mathrm{~s}^{-1}$ \\
$\mathrm{~A}-\mathrm{P}+\mathrm{B} \stackrel{k_{B}}{\longrightarrow} \mathrm{A}+\mathrm{B}-\mathrm{P}$ & CheB phosphorylation & $15 \mu \mathrm{M}^{-1} \mathrm{~s}^{-1}$ \\
$\mathrm{Y}-\mathrm{P}+\mathrm{Z} \underset{k_{1}}{\stackrel{k_{2}}{\rightleftharpoons}} \mathrm{Y}-\mathrm{P} \cdot \mathrm{Z}$ & CheY-P/CheZ association & $5 \mu \mathrm{M}^{-1} \mathrm{~s}^{-1}\left(0.5 \mathrm{~s}^{-1}\right)$ \\
$\mathrm{Y}-\mathrm{P} \stackrel{k_{3}}{\longrightarrow} \mathrm{Y}$ & CheY-P dephosphorylation & $200 \mathrm{~s}^{-1}$ \\
$\mathrm{~B}-\mathrm{P} \stackrel{k_{-B}}{\longrightarrow} \mathrm{B}$ & CheB-P dephosphorylation & $1.35 \mathrm{~s}^{-1}$ \\
\hline
\end{tabular}

representation of the signalling cascade within an E. coli cell than previously considered in analysing FCD in E. coli chemotaxis [35].

The model takes a MWC description of receptor clustering [24] and integrates this with the signalling pathway detailed in Table 1 . The MWC model uses a statistical mechanics based approach in order to describe the activity $(\Phi)$ of a receptor signalling team. Another way to think about the signalling team activity is that it represents the probability that the team will be in an active state at any one time. Receptor clusters are considered to consist of receptor teams consisting of Tar and Tsr receptors in the ratio $\nu_{a}: \nu_{s}$ where the subscripts $a / s$ denote Tar or Tsr receptors, respectively. The receptor signalling team activity may then be defined as

$$
\Phi=\frac{1}{1+e^{F}},
$$

in which $F$ represents the free-energy of a signalling team and is given by

$$
F=N\left[h(m)+f_{a}+f_{s}\right],
$$

where

$$
f_{a}=\nu_{a} \ln \left(\frac{1+[L] / K_{a}^{o f f}}{1+[L] / K_{a}^{o n}}\right),
$$

is the free-energy of the Tar receptors and

$$
f_{s}=\nu_{s} \ln \left(\frac{1+[L] / K_{s}^{o f f}}{1+[L] / K_{s}^{o n}}\right) .
$$

is the free-energy of the Tsr receptors. Here $[L]$ represents the extracellular ligand concentration, $m$ the receptor methylation level, $N$ denotes the number of receptors in a signalling team and $K_{a / s}^{o n / o f f}$ are the ligand dissociation constants for Tar $(a)$ and Tsr $(s)$ receptors in their active or inactive states, respectively. The function $h(m)$ represents the contribution from a receptors methylation level towards the total free-energy of a receptor (often called the "offset energy"). The form of this function is discussed in further detail in Section 3.1. Clausznitzer et al. [5] take $N=a_{0}+a_{1}[L]_{0}$, where $a_{0}$ and $a_{1}$ are fitting parameters and $[L]_{0}$ is an initial ligand concentration. We consider $N$ to be constant and discuss the implications when it depends upon the initial ligand concentration in Section 4.2.

Two key components of the adaptation process are the methylation and demethylation of receptors [17]. These two key components are represented by the kinetics of the average methylation level of a receptor in a cluster $m[5]$, such that

$$
\frac{d m}{d \tau}=\gamma_{R}(1-\Phi)-\gamma_{B}\left[b_{p}\right]^{2} \Phi
$$

in which $\gamma_{R}$ is the rate of methylation by CheR, $\gamma_{B}$ denotes the rate of demethylation by CheB-P and $\left[b_{p}\right]$ is the concentration of CheB-P. 
The model is completed by applying the law of mass action to the reactions in Table 1. For analysis purposes we follow the non-dimensionalisation stated by Clausznitzer et al. [5]. This yields the following system of nonlinear ODEs

$$
\begin{aligned}
\frac{d\left[a_{p}\right]}{d \tau} & =\Phi \cdot\left(1-\left[a_{p}\right]\right)-\alpha_{1} \bar{\kappa}_{Y}\left(1-\left[y_{p}\right]\right)\left[a_{p}\right]-\alpha_{2} \bar{\kappa}_{B}\left(1-\left[b_{p}\right]\right)\left[a_{p}\right] \\
\frac{d\left[y_{p}\right]}{d \tau} & =\bar{\kappa}_{Y}\left(1-\left[y_{p}\right]\right)\left[a_{p}\right]-\bar{\kappa}_{1}\left(\alpha_{3}-\left[y_{p} z\right]\right)\left[y_{p}\right]+\bar{\kappa}_{2}\left[y_{p} z\right] \\
\frac{d\left[y_{p} z\right]}{d \tau} & =\bar{\kappa}_{1}\left(\alpha_{3}-\left[y_{p} z\right]\right)\left[y_{p}\right]-\left(\bar{\kappa}_{2}+\bar{\kappa}_{3}\right)\left[y_{p} z\right] \\
\frac{d\left[b_{p}\right]}{d \tau} & =\bar{\kappa}_{B}\left(1-\left[b_{p}\right]\right)\left[a_{p}\right]-\bar{\kappa}_{-B}\left[b_{p}\right]
\end{aligned}
$$

with initial conditions

$$
\begin{gathered}
{\left[a_{p}\right]=a_{p}^{*}, \quad\left[y_{p}\right]=y_{p}^{*}, \quad\left[y_{p} z\right]=y_{p} z^{*},} \\
{\left[b_{p}\right]=b_{p}^{*} \text { and } m=m^{*},}
\end{gathered}
$$

where $^{*}$ denotes a steady-state value, each [...] denotes the concentration of the relevant protein or complex, the subscript $p$ indicates we are referring to the phosphorylated form of the protein, $\bar{\kappa}_{i}$ $(i=Y, B, 1,2,3,-B)$ are the rate constants of the reactions detailed in Table 2 and the $\alpha_{i}(i=1,2,3)$ are the total concentration ratios.

\subsection{The methylation function $h(m)$}

In order to proceed to determining whether this model is able to exhibit FCD we must define a representation of $h(m)$. This represents the "offset energy" [12] and is essentially the methylationdependent part of a receptor's free-energy. Within the literature there have been a number of forms taken for this function $[35,5,27]$ and we assess each against available experimental data. form

The models of Tu et al. [35], Clausznitzer et al. [5] and Shimizu et al. [27] all take the same basic

$$
h(m)=\alpha\left(m_{0}-m\right) .
$$

In this expression $\alpha$ is a scaling value and $m_{0}$ represents an initial methylation level (in the absence of any ligand). Tu et al. [35] takes $\alpha=2$ and $m_{0}=1$ to give

$$
h(m)=2(1-m),
$$

whilst Clausznitzer et al. [5] consider $\alpha=1 / 2$ and $m_{0}=2$, i.e.

$$
h(m)=1-\frac{m}{2}
$$

and Shimizu et al. [27] take $\alpha=2$ and $m_{0}=1 / 2$, giving

$$
h(m)=2\left(\frac{1}{2}-m\right)
$$

Each of these functional forms can be compared to experimental data $[8,27,36]$.

The experimental data of both Endres et al. [8] and Vaknin and Berg [36] use similar experimental techniques, whereby Tar receptors were genetically engineered to have either a glutamate (E) or a glutamine $(\mathrm{Q})$ at each of their four methylation sites $[8,36]$. In experiments such as these, a glutamine has similar functionality to a methylated glutamate [6]. Each data set considers slightly different combinations of modification states with the first set considering Tar receptors in QQQQ, QEQQ, QEQE and QEEE states [8]. The other data set gives results for Tar receptors in QQQQ, QEQQ, QEEE and EEEE states $[8,36]$. In order to make a comparison between the representations of $h(m)$ and the experimental data for amidation modifications it is necessary to convert them into the same form since one source data for individual receptors and the other for receptor dimers. This means the 


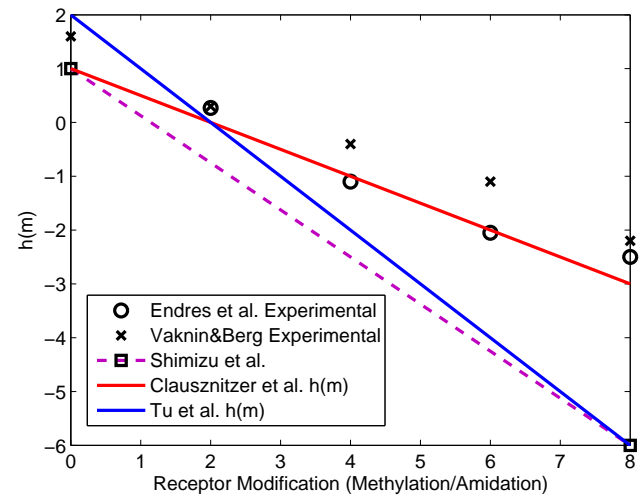

Fig. 3 A plot showing experimental and model data for the "offset energy". Experimental data for amidation modifications is from Endres et al. [8] (crosses) as well as Vaknin and Berg [36] (circles). Data for methylation modifications from Shimizu et al. [27] is also displayed (squares). Solid lines show the model data for the forms of $h(m)$ displayed in equations (14)-(16). (Color figure online)

Vaknin and Berg [36] data set refers to QEEE as equivalent to a methylation level of $m=1$ whereas the other would consider this as $m=2$. This requires a simple scaling of the $m$ values used for Figure 3 .

It is clear upon inspection of Figure 3 that the two data sets are similar and a good fit to the data will produce a model with a good level of realism. Examination of the choices of $h(m)$ reveals that the representation considered by Clausznitzer et al. [5] produces a better fit to the two sets of amidation modification data. Since the mathematical model of Clausznitzer et al. [5] has been shown to give a good fit to FRET experimental data, we adopt the form of $h(m)$ defined by equation (15) for the remainder of this manuscript. It is worth noting however that since each choice is a linear function, they should each be able to yield FCD behaviour, as per the conditions given by Lazova et al. [16].

\section{FCD in a MWC model of $E$. coli chemotaxis}

It is common in modelling chemotactic systems to only consider a response to a single chemoattractant. In much of the literature the chemoattractant of choice is $\alpha$-methylaspartate (MeAsp) due to the fact that it is non-metabolisable. We consider the effect of only binding MeAsp, for which it is known that Tar and Tsr receptor types are able to bind, the latter with a much lower affinity [5]. We are hence able to neglect the effects of MeAsp binding to Tsr receptors within the concentration range considered $[22]$. This feature allows us to re-write equation (6) as

$$
F=N\left[\left(1-\frac{m}{2}\right)+\nu_{a} \ln \left(\frac{1+[L] / K_{a}^{o f f}}{1+[L] / K_{a}^{o n}}\right)\right] .
$$

A key assumption from the literature in showing the existence of FCD in a system of this type is that $K_{a}^{o f f} \ll[L] \ll K_{a}^{o n}$, i.e. the extracellular ligand concentration must be much larger than the ligand dissociation constant of an inactive receptor while also being much smaller than the dissociation constant for an active receptor. This is a sufficient condition for FCD, however further conditions for a model of this form are discussed in section 5.1. Following Shoval et al. [29] this allows us to make two simplifications to the logarithmic terms in equation (6), namely

$$
\ln \left(1+\frac{[L]}{K_{a}^{o n}}\right) \approx \ln (1)=0
$$

and

$$
\ln \left(1+\frac{[L]}{K_{a}^{o f f}}\right) \approx \ln \left(\frac{[L]}{K_{a}^{o f f}}\right)
$$

which reduces equation (17) to

$$
F=N\left[\left(1-\frac{m}{2}\right)+\nu_{a} \ln \left(\frac{[L]}{K_{a}^{o f f}}\right)\right] .
$$


Substituting this expression into equation (5), we obtain

$$
\Phi=\frac{1}{1+\left(\frac{[L]}{x(m)}\right)^{N \nu_{a}}}
$$

in which $x(m)=K_{a}^{o f f} \exp \left((m-2) / 2 \nu_{a}\right)$.

It is now possible to assess whether the model considered here will satisfy the sufficient conditions for FCD $[29,28]$. These conditions only apply to two expressions which may appear to be problematic given the size of the model considered here. The composition of the model, however, means that it is only two expressions (those for $\Phi$ and $x(m)$ ) that must be taken into account when attempting to show the existence of FCD.

In order to examine the first sufficient condition given by equation (3) we must consider the expression for $x(m)$. This is the effective dissociation constant of the receptor to the chemoattractant [29]. Testing this condition requires us to examine the temporal derivative of $x(m)$

$$
\begin{aligned}
\dot{x} & =\frac{1}{2 \nu_{a}} x(m) \frac{d m}{d \tau} \\
& =f(x(m), \Phi,[L]) .
\end{aligned}
$$

Testing of the sufficient condition given by equation (3) is carried out by multiplying $x(m)$ and $[L]$ by some constant $p>0$

$$
f(p x(m), \Phi, p[L])=\frac{1}{2 \nu_{a}} p x(m) \frac{d m}{d \tau}=p\left(\frac{1}{2 \nu_{a}} x(m) \frac{d m}{d \tau}\right)=p f(x(m), \Phi,[L]),
$$

which clearly satisfies equation (3).

Examination of equation (21) is necessitated by condition (4). Noticing that

$$
\Phi=\frac{1}{1+\left(\frac{[L]}{x(m)}\right)^{N \nu_{a}}}=g(x(m), \Phi,[L])
$$

allows this condition to be tested in a similar manner to equation (3). Multiplication of the $x(m)$ and $[L]$ terms by $p$ yields

$$
g(p x(m), \Phi, p[L])=\frac{1}{1+\left(\frac{p[L]}{p x(m)}\right)^{N \nu_{a}}}=\frac{1}{1+\left(\frac{[L]}{x(m)}\right)^{N \nu_{a}}}=g(x(m), \Phi,[L]) .
$$

As with the first homogeneity condition this gives clear evidence that the sufficient condition (4) holds.

We have clearly shown that both of the homogeneity conditions hold for the model. There are, however, four sufficient conditions required for the existence of FCD $[29,28]$. It has been stated that a model of the form defined by equations (5), (20), (7)-(11) exhibits exact adaptation [5]. The system was also found to be stable upon inspection of the eigenvalues of the stability matrix. Having satisfied all four sufficient conditions we expect each protein and complex within the model to exhibit FCD since the ODEs for each chemotaxis proteins and complex are not ligand dependent in the range $K_{a}^{o f f} \ll[L] \ll K_{a}^{o n}$.

\subsection{Numerical simulations}

We now use numerical simulations to provide examples of the model exhibiting FCD. The model equations were solved in MATLAB (MathWorks) using the ordinary differential equation solver ode15s. The production of numerical simulations necessitates the definition of a full parameter set as detailed in Table 2.

Simulations were run by first obtaining model steady-states for each protein and protein complex given a specific ligand concentration. We note that each of the protein concentrations as well as the 
Table 2 Non-dimensional parameter values used to create numerical simulations. ${ }^{*}$ indicates that these values were taken from the Bray Group data website [3]. All other parameters were calculated using values stated by Clausznitzer et al. [5].

\begin{tabular}{lll}
\hline Symbol & Definition & Value \\
\hline $\bar{\kappa}_{Y}$ & Rate of phosphotransfer to CheY from CheA-P & 79 \\
$\bar{\kappa}_{B}$ & Rate of phosphotransfer to CheB from CheA-P & 11.85 \\
$\bar{\kappa}_{-B}$ & CheB-P autodephosphorylation rate constant & 0.135 \\
$\bar{\kappa}_{1}$ & CheY-P/CheZ association rate constant & 4.85 \\
$\bar{\kappa}_{2}$ & CheY-P/CheZ dissociation rate constant & 0.05 \\
$\bar{\kappa}_{3}$ & CheY-P dephosphorylation rate constant & 20 \\
$\alpha_{1}$ & Ratio of total CheY to total CheA & 1.228 \\
$\alpha_{2}$ & Ratio of total CheB to total CheA & $3.5 \times 10^{-2}$ \\
$\alpha_{3}$ & Ratio of total CheZ to total CheY & 0.392 \\
$\gamma_{R}$ & Rate constant for methylation by CheR & $6 \times 10^{-4}$ \\
$\gamma_{B}$ & Rate constant for demethylation by CheB-P & $2.46 \times 10^{-2}$ \\
$\nu_{a}$ & Ratio of Tar:Tsr receptors $\left(\nu_{a}: \nu_{s}\right)$ & 1 \\
$K_{a}^{o f f}$ & Ligand dissociation constant of inactive Tar receptors & $2 \times 10^{-2} \mathrm{mM}$ \\
$N$ & Number of receptors in a signalling team & 18 \\
{$\left[A_{t o t}\right]$} & Total concentration of CheA protein & $7.9 \mu \mathrm{M}^{*}$ \\
{$\left[B_{t o t}\right]$} & Total concentration of CheB protein & $0.28 \mu \mathrm{M}^{*}$ \\
{$\left[Y_{t o t}\right]$} & Total concentration of CheY protein & $9.7 \mu \mathrm{M}^{*}$ \\
{$\left[Z_{t o t}\right]$} & Total concentration of CheZ protein & $3.8 \mu \mathrm{M}^{*}$ \\
\hline
\end{tabular}

receptor cluster activity have steady-states that are independent of the extracellular ligand concentration $[L]$. This can be seen from the steady-state of the receptor activity, equation (7), which is given by

$$
\Phi^{*}=\frac{1}{1+\frac{\gamma_{B}}{\gamma_{R}}\left[b_{p}\right]^{* 2}},
$$

where * indicates a steady-state. Substitution of equation (26) into equations (8)-(11) allows us to solve a series of four coupled algebraic equations to obtain $\left[a_{p}\right]^{*},\left[b_{p}\right]^{*},\left[y_{p}\right]^{*}$ and $\left[y_{p} z\right]^{*}$. The steady-state for the receptor methylation level (using equations (20) and (26)), does depend on the ligand concentration and is given by

$$
m^{*}=2\left[1-\ln \left(\left(\frac{\gamma_{B}}{\gamma_{R}}\left[b_{p}\right]^{* 2}\right)^{1 / N}\left(\frac{K_{a}^{o f f}}{[L]}\right)\right)\right] .
$$

In the range of ligand concentrations considered for our numerical simulations $(0.1 \mathrm{mM} \leq[L] \leq 0.7 \mathrm{mM})$ and receptor clusters of size $N \gg 1$, this gives

$$
m^{*} \approx 2\left[1-\ln \left(\frac{K_{a}^{o f f}}{[L]}\right)\right],
$$

meaning that the methylation level is dominated by the ratio of $K_{a}^{o f f}$ to $[L]$. It is clear that in cases considered here, in which we have $K_{a}^{\text {off }} \ll[L]$, this will yield a fairly large steady-state methylation level. It is often stated that each Tar receptor dimer possesses eight methylation sites [12]. At first glance this would seem to make our high methylation levels seem unrealistic. This is not the case as each CheR and CheB-P is able to bind to a 35 amino-acid tether attached to a Tar receptor [2]. Once bound to this tether, each CheR or CheB-P is able to methylate/demethylate approximately six nearby receptor dimers [9]. This group of receptor dimers is termed an assistance neighbourhood [18]. The effect of these assistance neighbourhoods is to increase the ladder of possible methylation states from 8 to $\sim 48$ [9], meaning that high methylation levels, as defined by equation (28), are feasible.

A number of numerical simulations were used to test for FCD in the range of initial ligand concentrations $0.1 \mathrm{mM} \leq[L]_{0} \leq 0.7 \mathrm{mM}$. Figures 4 and 5 show the behaviour of each chemotaxis protein, complex and the receptor methylation level in response to two and five fold changes in ligand concentration for initial ligand concentrations of $0.1 \mathrm{mM}$ and $0.2 \mathrm{mM}$. From the results in Figures 4 and 5 FCD is evident for each chemotaxis protein and the respective complex; each shows the same shape, amplitude and recovery time in response to equal fold changes in ligand concentration. 

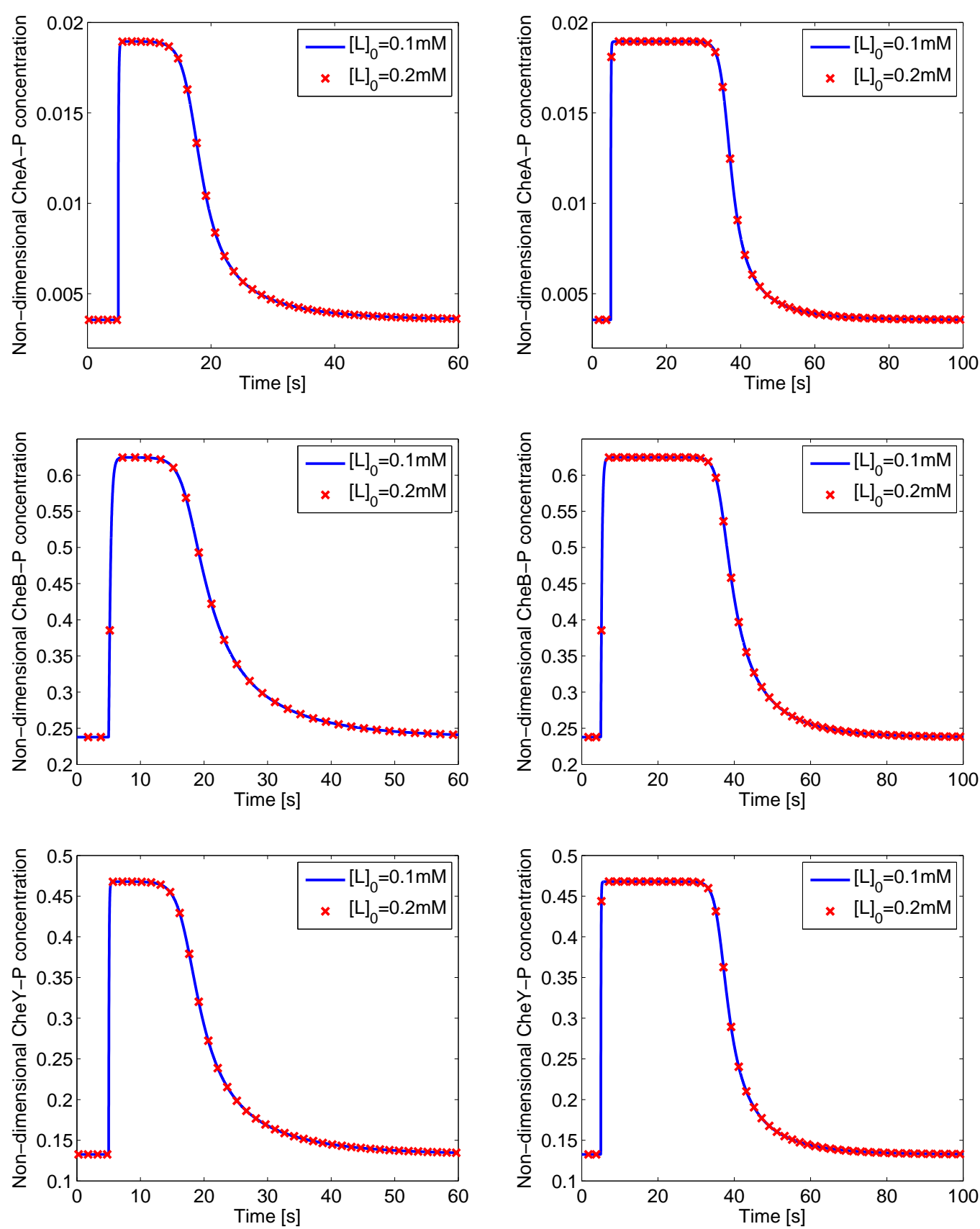

Fig. 4 Plots displaying the behaviour of each chemotaxis protein in response to two fold (left column) and five fold (right column) changes in extracellular ligand concentration. Blue lines represent the response for an initial ligand concentration of $0.1 \mathrm{mM}$ and red crosses for an initial ligand concentration of $0.2 \mathrm{mM}$. (Color figure online)

As mentioned in Section 4, we have made a number of simplifying assumptions to the original model proposed by [5]. In spite of these assumptions the model still gives good agreement with experimental FRET measurements [5]. We note in particular the similarity between model and experiment in the time taken for the system to fully adapt to a change and the amplitude and profile of the response curves obtained. 

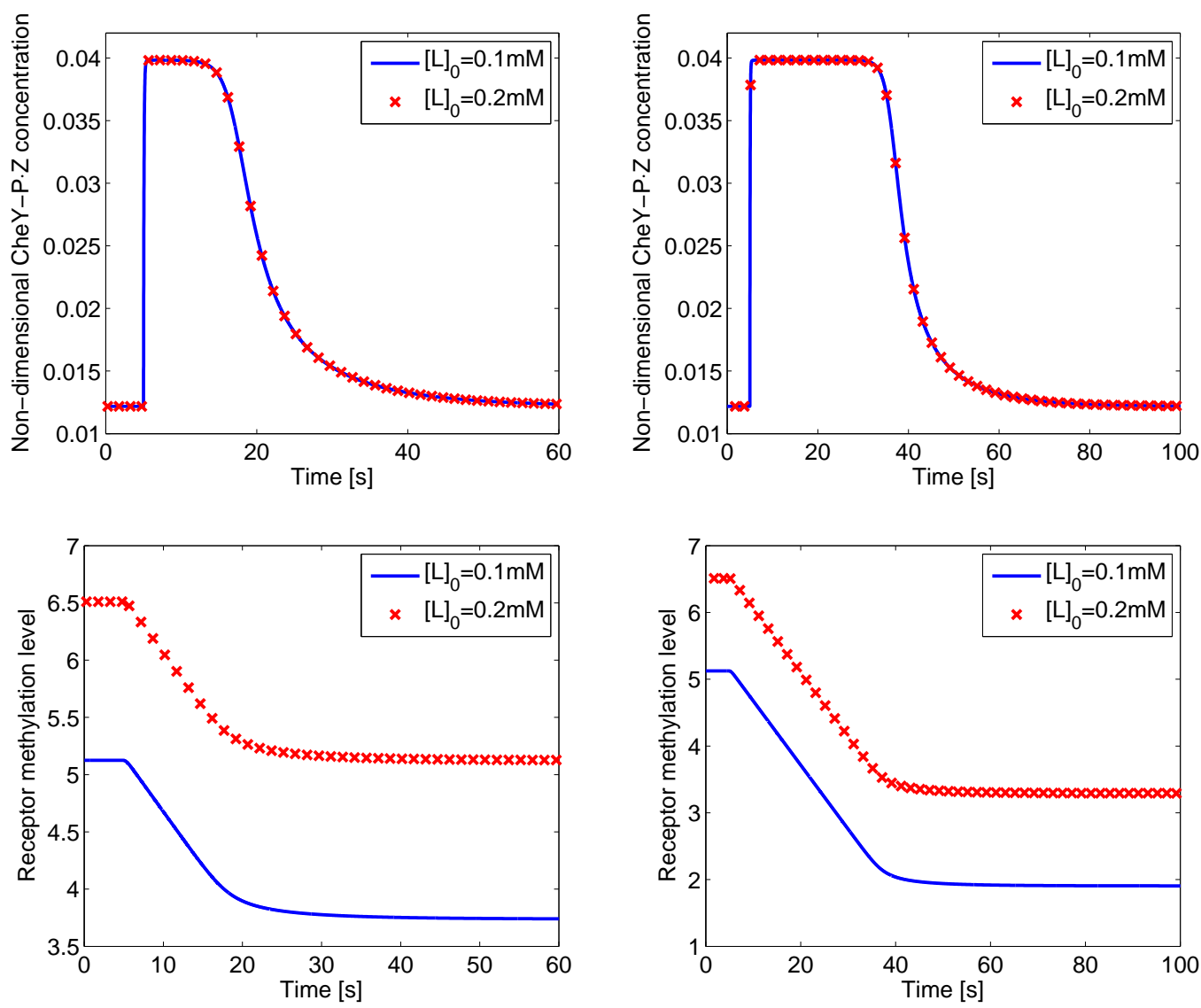

Fig. 5 Plots displaying the behaviour of the CheY-P/CheZ complex and average receptor methylation level of a signalling team in response to two fold (left column) and five fold (right column) changes in extracellular ligand concentration. Blue lines represent the response for an initial ligand concentration of $0.1 \mathrm{mM}$ and red crosses for an initial ligand concentration of $0.2 \mathrm{mM}$. (Color figure online)

\subsection{Parameter sensitivity analysis}

The results of Section 4 suggest that FCD for each of the proteins and respective complex will be independent of any variation in parameters associated with the signalling cascade. To test this result we undertook a local sensitivity analysis of the kinetic rate parameters and protein concentrations. Each of the parameter values in Table 2 were both increased and decreased by an order of magnitude such that $0.1 x \leq x \leq 10 x$, where $x$ represents the initial parameter value. Results showed that FCD still held for all elements of the signalling cascade irrespective of which parameter value was varied, thus confirming the theoretical predictions.

Interestingly, there is experimental evidence that the size of a receptor signalling team varies with the extracellular ligand concentration [8]. With this being the case Clausznitzer et al. [5] state an expression for the size of a receptor signalling team, which is

$$
N=a_{0}+a_{1}[L]_{0},
$$

where $a_{0}$ and $a_{1}$ are fitting parameters (given values of $a_{0}=17.5$ and $a_{1}=3.35$, respectively) and $[L]_{0}$ represents the initial extracellular ligand concentration. Consideration of a model such as this would result in the use of a different value of $N$ for each simulation. Essentially this would amount to changing the value of a parameter between consecutive runs of the model. Based on the theory presented in previous sections we would expect that this would not result in FCD, a prediction which is verified by Figure (6). Given the dependence of the signalling team size $N$ on the initial ligand concentration a small variation was observed between responses for the same fold change. In order to 
Table 3 Dissociation constants for active and inactive Tar receptors, as reported in the literature.

\begin{tabular}{lll}
\hline \multicolumn{3}{c}{ Dissociation Constant } \\
Inactive Receptor $\left(K_{a}^{\text {off }}\right)$ & Active Receptor $\left(K_{a}^{\text {on }}\right)$ & Source \\
\hline$\sim 18 \mu \mathrm{M}$ & $\sim 3000 \mu \mathrm{M}$ & {$[35,22,13]$} \\
$20 \mu \mathrm{M}-25 \mu \mathrm{M}$ & $500 \mu \mathrm{M}$ & {$[5,21,12,15,9,11,7]$} \\
$30 \mu \mathrm{M}$ & $1000 \mu \mathrm{M}$ & {$[30]$} \\
$1.7 \mu \mathrm{M}$ & $12 \mu \mathrm{M}$ & {$[25,26]$} \\
\hline
\end{tabular}

quantify this variation over the two regimes of FCD behaviour we consider a two fold increase from initial ligand concentrations corresponding to the end points of each FCD regime. In the range of ligand concentrations defining the two experimentally determined regimes of FCD [16] this variation is rather small ( $<5 \%$ of the initial CheY-P response magnitude for two fold changes in ligand concentration, for both the FCD1 and FCD2 regimes). We would expect this variation to be undetectable experimentally.

\section{How realistic is $K_{a}^{o f f} \ll[L] \ll K_{a}^{o n}$ ?}

The results of the previous sections required us to make the assumption that $K_{a}^{o f f} \ll[L] \ll K_{a}^{o n}$ in order for the model to follow the FCD conditions of equations (3) and (4).

In order to see if this condition would hold for all cases in vivo we conducted a literature search and obtained dissociation constant values for Tar receptors, as detailed in Table 3 . It is clear that these values vary significantly depending on the source. A group of papers by Tu and colleagues use values that are clearly compatible with the condition $K_{a}^{o f f} \ll[L] \ll K_{a}^{o n}$. Sourjik and Berg have also utilised values which give a reasonably good agreement with this condition, albeit with a smaller range capable of producing FCD. It is unlikely that we can say FCD holds using the range proposed by Endres, Wingreen and colleagues. The reason for this is the value for $K_{a}^{o n}$ which is given as $500 \mu \mathrm{M}$. This is a fair amount larger than the values for $K_{a}^{o f f}$, however it is unlikely to be a wide enough separation to allow for FCD. The final range was proposed by Bray and colleagues who suggest that the two dissociation constants should be very close together. This will clearly make it difficult to obtain FCD from a model using these values.

The range of values used for the dissociation constants is clearly very large. This makes an assessment on the feasibility of the condition $K_{a}^{o f f} \ll[L] \ll K_{a}^{o n}$ always holding very difficult to make. As such we defer judgement until there is further experimental evidence in support of one set of values in Table 3 .

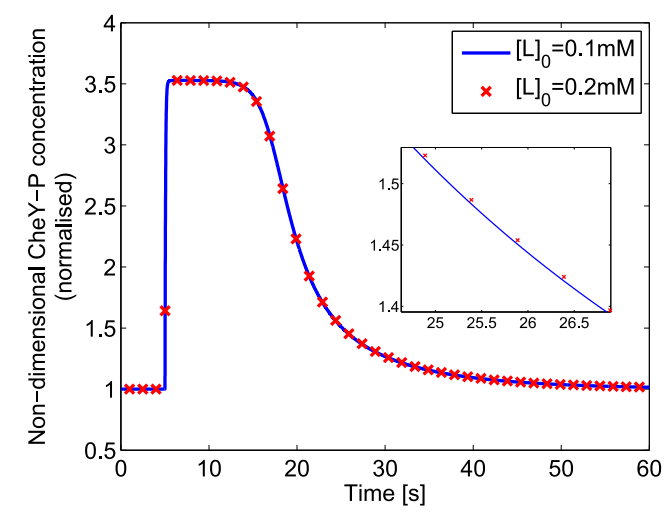

Fig. 6 FCD does not hold when considering the signalling team size expression $N=a_{0}+a_{1}[L]_{0}$. Simulations are displayed for initial ligand concentrations of $0.1 \mathrm{mM}$ and $0.2 \mathrm{mM}$ in response to a two fold change in ligand concentration, normalised with respect to the initial steady-state concentration. The inset shows more clearly that FCD does not hold since the shape of the response is not the same in both simulations. (Color figure online) 
Table 4 Dissociation constants of Tar and Tsr receptors for MeAsp, as reported in the literature.

\begin{tabular}{llll}
\hline Type & $K_{a / s}^{o f f}$ & $K_{a / s}^{o n}$ & Source \\
\hline $\operatorname{Tar}(a)$ & $1.7 \times 10^{-3} \mathrm{mM}$ & $0.5 \mathrm{mM}$ & {$[5,25]$} \\
$\operatorname{Tsr}(s)$ & $100 \mathrm{mM}$ & $10^{6} \mathrm{mM}$ & {$[5]$} \\
\hline
\end{tabular}

\subsection{Multiple receptor types}

We have so far assumed that only Tar receptors are able to detect the chemoattractant gradient. It is unlikely in vivo that a cell will produce a response to MeAsp using only one receptor type given Tsr receptors are able to bind MeAsp, albeit with a much lower affinity [5]. As such we now consider whether it is feasible that a cell could exhibit FCD in the case where two or more receptor types can detect the same attractant gradient.

Theoretically it can be shown that FCD will hold for a multiple receptor model of the form of equation (17); one considers the conditions detailed in equations (3) and (4) for a generalised function $\varphi(p, x)$ [29] of the form $\varphi=p^{R}$ (A. Hamadeh and E. Sontag, personal communication), where $R$ represents the sum of all $\nu_{i}$ for each receptor type $i(i=a, s, \ldots)$. We undertook numerical simulations of our model using this assumption with the receptor free-energy described by equation (17) and found that all proteins and complexes in the signalling network still demonstrated FCD as shown in Figure 8.

Recent experimental work has shown FCD may be observed in cells expressing a wild-type receptor configuration [16]. This same work demonstrated the existence of two regimes of FCD behaviour, namely FCD1 $\left([L]_{0}=0.018-0.23 \mathrm{mM}\right)$ and FCD2 $\left([L]_{0}=0.82-10.3 \mathrm{mM}\right)$, where $[L]_{0}$ is an initial MeAsp concentration. In light of this and the results displayed in Figure 8 we conducted a search of the literature to see if these two FCD regimes may be explained theoretically. The search yielded values of the dissociation constants of MeAsp binding for both Tar and Tsr receptors, as shown in Table 4 . Theoretically, it is clear upon inspection of these values that it would be difficult in the case where multiple receptors bind the same ligand to choose a ligand concentration that would ensure $K_{i}^{o f f} \ll$ $[L] \ll K_{i}^{o n}$ would hold for each receptor type. However, the conditions $[L] \ll K_{i}^{\text {off }} \ll K_{i}^{\text {on }}$ and $K_{i}^{\text {off }} \ll$ $K_{i}^{o n} \ll[L]$ may also result in similar receptor free-energy expressions to those given by equations (18) and (19). Hence, FCD may potentially be found even in cases where $K_{i}^{\text {off }} \ll[L] \ll K_{i}^{\text {on }}$ does not hold. In particular, we may use the values in Table 4 to explain the two regimes of FCD behaviour. The FCD1 regime $\left([L]_{0}=0.018-0.23 \mathrm{mM}\right)$ clearly satisfies $K^{\text {off }} \ll[L] \ll K^{\text {on }}$ for Tar receptors and

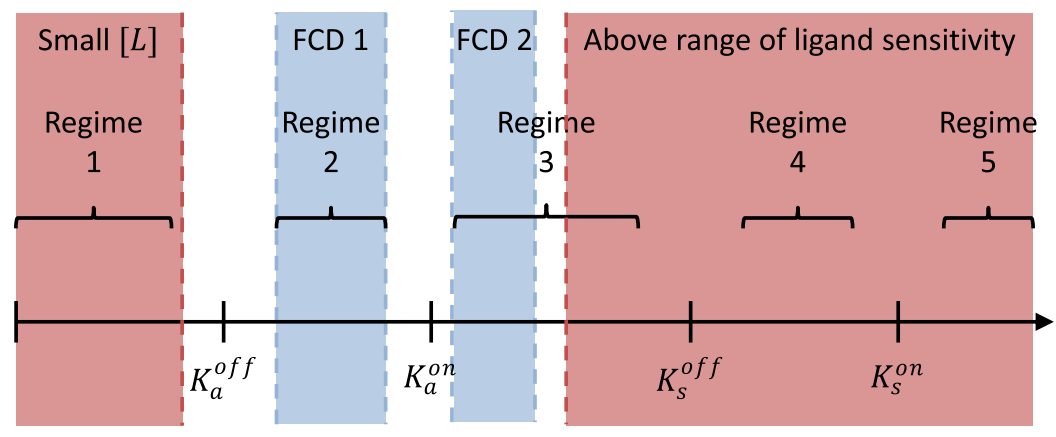

$[L](m M)$

Fig. 7 For a cell with two receptor types capable of sensing the same ligand we would predict five separate regimes of FCD behaviour (braces). Experimental work was able to demonstrate just two regimes of FCD behaviour, likely to be due to the cells range of ligand sensitivity and small ligand concentrations not considered experimentally (red boxes). As such, we have two remaining regimes of FCD behaviour, namely FCD1 and FCD2 (blue boxes). Note that the regime FCD2 is truncated somewhat by the ligand sensitivity range of the cell. 
$[L] \ll K^{o f f} \ll K^{o n}$ for Tsr receptors, when binding MeAsp. Similarly, for the FCD2 regime $\left([L]_{0}=0.82-\right.$ $10.3 \mathrm{mM})$ we satisfy $K^{\text {off }} \ll K^{o n} \ll[L]$ for Tar receptors and $[L] \ll K^{o f f} \ll K^{o n}$ for Tsr receptors. In fact, theoretically we would predict that for $n$ receptors with well separated dissociation constants for the binding of one ligand, there would be $2 n+1$ different FCD regimes, as shown in Figure 7 . This should be the case assuming that there was an infinite range of receptor sensitivity to the relevant ligand. As mentioned earlier, the experimental evidence suggests just two FCD regimes [16]. Based on our work, we could predict 5 different FCD regimes for $E$. coli, however the lowest ligand concentration regime is likely to be very small and thus not observed missed experimentally. The other two predicted FCD regimes not found are likely to be due to the restricted ligand sensitivity range of the receptors (see Figure 7).

In light of this we consider the effect of the cell detecting two ligands (MeAsp and serine). This situation is of interest since the affinity of Tsr receptors to MeAsp is so low, meaning MeAsp binding to Tsr receptors has generally very little effect. In this case we consider a situation whereby both receptor types are present in similar numbers and bind separate ligands. Parameter values have already been given for the binding of MeAsp to Tar receptors in Table 2. For this particular case we consider the ligand (serine) dissociation constant of an inactive Tsr receptor to be $K_{s}^{o n}=6 \times 10^{-3} \mathrm{mM}$ [14]. As per Kalinin et al. [14] we consider receptors to be present in different quantities, namely $N_{T a r}=6$ and $N_{T s r}=12$, where $N_{T a r}$ is the number of Tar receptors and $N_{T s r}$ is the number of Tsr receptors present in a signalling team.

We consider a different fold change for each ligand in addition to the case where each ligand is subject to an equal fold change. As with the previous multiple receptor case, we discover that FCD will hold when each receptor satisfied either $[L] \ll K_{i}^{\text {off }} \ll K_{i}^{\text {on }}, K_{i}^{\text {off }} \ll[L] \ll K_{i}^{\text {on }}$ or $K_{i}^{o f f} \ll K_{i}^{o n} \ll[L]$. Figure 9 displays numerical results verifying that FCD holds in this signalling team configuration. It should be feasible given experimentally determined values for the ligand dissociation constants of Tar and Tsr receptors to MeAsp and serine in both cases where receptors bind either the same or separate ligands for the behaviour to be observed.

\section{Summary and conclusions}

FCD has been shown to hold in a whole-pathway model of intracellular signalling in E. coli chemotaxis formulated by Clausznitzer et al. [5]. A number of assumptions were made in ensuring the model would fit with the criteria for an FCD response. This results in a reduced form of the model that shows good agreement with experimental data [5] in terms of time scales and response amplitudes.

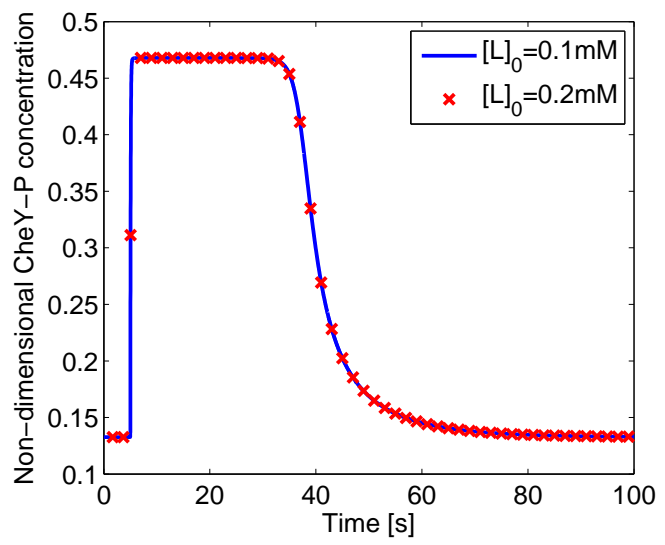

(a) CheY-P

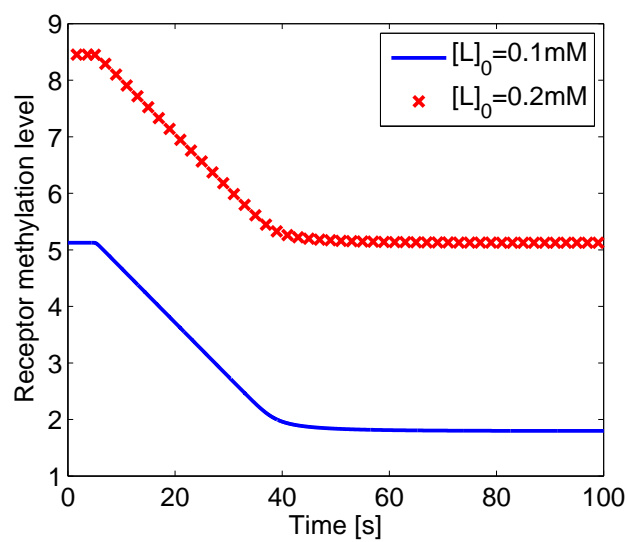

(b) Receptor methylation

Fig. 8 Theoretically E. coli cells are able to display FCD in the presence of two receptor types. Plots displayed here are for a model containing both Tar and Tsr receptors. Responses are for a two fold change in extracellular ligand concentration with initial concentrations of $0.1 \mathrm{mM}$ and $0.2 \mathrm{mM}$. (a) shows the response of CheY-P and (b) the receptor methylation level. Plots were created using $K_{s}^{o f f}=0.1 \mathrm{mM}$ and $\nu_{s}=1.4$. 


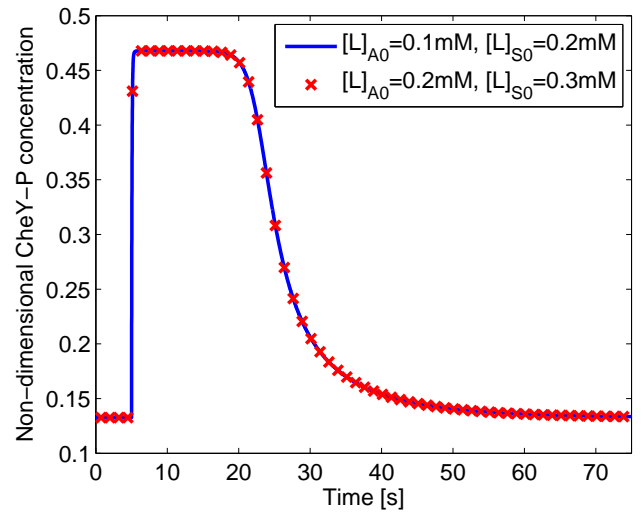

Fig. 9 FCD may be displayed in situations where multiple receptor types act to bind separate ligands. Responses are for initial ligand concentrations of $[L]_{a 0}=0.1 \mathrm{mM}$ with $[L]_{s 0}=0.2 \mathrm{mM}$ and $[L]_{a 0}=0.2 \mathrm{mM}$ with $[L]_{s 0}=0.3 \mathrm{mM}$. Ligand concentration changes were two-fold in MeAsp and three-fold in serine.

A previous theoretical study states that in order for FCD to hold, the parameter values used within the mathematical model should remain constant. As such, we would expect FCD to be a robust property of the cell signalling cascade, i.e. the precise value of each parameter is not important for FCD to hold so long as the value remains constant. Within this work a parameter sensitivity analysis shows this to be the case. We do however note that considering a model whereby receptor signalling team size is dependent on the ambient ligand concentration such as that considered by Clausznitzer et al. [5] causes FCD to fail. Importantly though we find only a small variation from FCD behaviour within the two experimentally determined FCD regimes.

Recent experimental work has demonstrated the existence of two regimes of FCD behaviour. Here we have demonstrated that FCD may be observed in situations whereby multiple receptor types are considered. In particular, we found that for a cell with $n$ different receptor types there would potentially exist $2 n+1$ separate regimes of FCD behaviour. Within this work consideration of the conditions $[L] \ll K^{\text {off }} \ll K^{o n}, K^{\text {off }} \ll[L] \ll K^{o n}$ and $K^{\text {off }} \ll K^{o n} \ll[L]$ for a model comprising two receptor types able to sense the same ligand gives an explanation for the existence of the multiple regimes of FCD. This is then shown to be feasible based upon experimentally determined values of the ligand dissociation constants for each receptor type.

The results of our work raise interesting questions as to the occurrence of FCD in more complicated cell signalling architectures. Results here tend to suggest that so long as the sensing components of the cascade (most commonly those areas of the cell where receptor-ligand binding is controlled) can exhibit FCD then the underlying signalling cascade should not affect the FCD response and has interesting implications for other intracellular signalling cascades in prokaryotic and eukaryotic systems.

Acknowledgements MPE was supported by an Engineering and Physical Sciences Research Council Studentship, UK, and MJT by a Research Council UK Fellowship during the period in which this research was undertaken.

\section{References}

1. Baker, M., Wolanin, P., Stock, J.: Signal transduction in bacterial chemotaxis. Bioessays 28(1), 9-22 (2006)

2. Barnakov, A., Barnakova, L., Hazelbauer, G.: Efficient adaptational demethylation of chemoreceptors requires the same enzyme-docking site as efficient methylation. Proc. Natl. Acad. Sci. USA 96(19), 10,667 (1999)

3. Bray, D.: Bray research group data website. http://www.pdn.cam.ac.uk/groups/compcell/Data.html. Accessed: 11/02/2013

4. Bren, A., Welch, M., Blat, Y., Eisenbach, M.: Signal termination in bacterial chemotaxis: CheZ mediates dephosphorylation of free rather than switch-bound CheY. Proc. Natl. Acad. Sci. USA 93(19), 10,090 (1996)

5. Clausznitzer, D., Oleksiuk, O., Løvdok, L., Sourjik, V., Endres, R.: Chemotactic response and adaptation dynamics in Escherichia coli. PLoS Comput. Biol. 6(5), e1000,784 (2010)

6. Dunten, P., Koshland Jr, D.: Tuning the responsiveness of a sensory receptor via covalent modification. J. Biol. Chem. 266(3), 1491-1496 (1991) 
7. Endres, R., Falke, J., Wingreen, N.: Chemotaxis receptor complexes: from signaling to assembly. PLoS Comput. Biol. 3(7), e150 (2007)

8. Endres, R., Oleksiuk, O., Hansen, C., Meir, Y., Sourjik, V., Wingreen, N.: Variable sizes of Escherichia coli chemoreceptor signaling teams. Mol. Syst. Biol. 4(1) (2008)

9. Endres, R., Wingreen, N.: Precise adaptation in bacterial chemotaxis through "assistance neighborhoods". Proc. Natl. Acad. Sci. USA 103(35), 13,040-13,044 (2006)

10. Grebe, T., Stock, J.: Bacterial chemotaxis: the five sensors of a bacterium. Curr. Biol. 8(5), R154-R157 (1998)

11. Hansen, C., Endres, R., Wingreen, N.: Chemotaxis in Escherichia coli: a molecular model for robust precise adaptation. PLoS Comput. Biol. 4(1), e1 (2008)

12. Hansen, C., Sourjik, V., Wingreen, N.: A dynamic-signaling-team model for chemotaxis receptors in Escherichia coli. Proc. Natl. Acad. Sci. USA 107(40), 17,170-17,175 (2010)

13. Jiang, L., Ouyang, Q., Tu, Y.: Quantitative modeling of Escherichia coli chemotactic motion in environments varying in space and time. PLoS Comput. Biol. 6(4), e1000,735 (2010)

14. Kalinin, Y., Jiang, L., Tu, Y., Wu, M.: Logarithmic sensing in Escherichia coli bacterial chemotaxis. Biophys. J. 96(6), 2439-2448 (2009)

15. Keymer, J., Endres, R., Skoge, M., Meir, Y., Wingreen, N.: Chemosensing in Escherichia coli: two regimes of two-state receptors. Proc. Natl. Acad. Sci. USA 103(6), 1786-1791 (2006)

16. Lazova, M., Ahmed, T., Bellomo, D., Stocker, R., Shimizu, T.: Response rescaling in bacterial chemotaxis. Proc. Natl. Acad. Sci. USA 108(33), 13,870-13,875 (2011)

17. Li, G., Weis, R.: Covalent modification regulates ligand binding to receptor complexes in the chemosensory system of Escherichia coli. Cell 100(3), 357-365 (2000)

18. Li, M., Hazelbauer, G.: Adaptational assistance in clusters of bacterial chemoreceptors. Mol. Microbiol. 56(6), 1617-1626 (2005)

19. Lipkow, K.: Changing cellular location of CheZ predicted by molecular simulations. PLoS Comput. Biol. 2(4), e39 (2006)

20. Lipkow, K., Andrews, S., Bray, D.: Simulated diffusion of phosphorylated CheY through the cytoplasm of Escherichia coli. J. Bacteriol. 187(1), 45-53 (2005)

21. Meir, Y., Jakovljevic, V., Oleksiuk, O., Sourjik, V., Wingreen, N.: Precision and kinetics of adaptation in bacterial chemotaxis. Biophys. J. 99(9), 2766-2774 (2010)

22. Mello, B., Tu, Y.: Effects of adaptation in maintaining high sensitivity over a wide range of backgrounds for Escherichia coli chemotaxis. Biophys. J. 92(7), 2329-2337 (2007)

23. Mesibov, R., Ordal, G., Adler, J.: The range of attractant concentrations for bacterial chemotaxis and the threshold and size of response over this range. J. Gen. Physiol. 62(2), 203-223 (1973)

24. Monod, J., Wyman, J., Changeux, J.: On the nature of allosteric transitions: a plausible model. J. Mol. Biol. 12(7), 88-118 (1965)

25. Morton-Firth, C., Shimizu, T., Bray, D.: A free-energy-based stochastic simulation of the tar receptor complex. J. Mol. Biol. 286(4), 1059-1074 (1999)

26. Shimizu, T., Aksenov, S., Bray, D.: A spatially extended stochastic model of the bacterial chemotaxis signalling pathway. J. Mol. Biol. 329(2), 291-309 (2003)

27. Shimizu, T.S., Tu, Y., Berg, H.C.: A modular gradient-sensing network for chemotaxis in escherichia coli revealed by responses to time-varying stimuli. Mol. Syst. Biol. 6(1) (2010)

28. Shoval, O., Alon, U., Sontag, E.: Symmetry invariance for adapting biological systems. SIAM J. Appl. Dyn. Syst. 10, 857 (2011)

29. Shoval, O., Goentoro, L., Hart, Y., Mayo, A., Sontag, E., Alon, U.: Fold-change detection and scalar symmetry of sensory input fields. Proc. Natl. Acad. Sci. USA 107(36), 15,995-16,000 (2010)

30. Sourjik, V., Berg, H.: Functional interactions between receptors in bacterial chemotaxis. Nature 428(6981), 437-441 (2004)

31. Springer, W., Koshland, D.: Identification of a protein methyltransferase as the CheR gene product in the bacterial sensing system. Proc. Natl. Acad. Sci. USA 74(2), 533 (1977)

32. Stock, J., Koshland, D.: A protein methylesterase involved in bacterial sensing. Proc. Natl. Acad. Sci. USA $\mathbf{7 5}(8), 3659(1978)$

33. Tindall, M., Porter, S., Maini, P., Gaglia, G., Armitage, J.: Overview of mathematical approaches used to model bacterial chemotaxis i: the single cell. Bull. Math. Biol. 70(6), 1525-1569 (2008)

34. Tindall, M.J., Gaffney, E.A., Maini, P.K., Armitage, J.P.: Theoretical insights into bacterial chemotaxis. Wiley Interdiscip. Rev. Syst. Biol. Med 4(3), 247-259 (2012). DOI 10.1002/wsbm.1168. URL http://dx.doi.org/10.1002/wsbm.1168

35. Tu, Y., Shimizu, T., Berg, H.: Modeling the chemotactic response of Escherichia coli to time-varying stimuli. Proc. Natl. Acad. Sci. USA 105(39), 14,855 (2008)

36. Vaknin, A., Berg, H.: Physical responses of bacterial chemoreceptors. J. Mol. Biol. 366(5), 1416-1423 (2007)

37. Wadhams, G., Armitage, J.: Making sense of it all: bacterial chemotaxis. Nat. Rev. Mol. Cell Bio. 5(12), 1024-1037 (2004)

38. Welch, M., Oosawa, K., Aizawa, S., Eisenbach, M.: Phosphorylation-dependent binding of a signal molecule to the flagellar switch of bacteria. Proc. Natl. Acad. Sci. USA 90(19), 8787 (1993) 